Portland State University

PDXScholar

$11-18-2016$

\title{
A Picture is Worth a Thousand Words: a Content Analysis of Self-Inflicted Face-ism on Instagram
}

Madeline Powell

Portland State University

Follow this and additional works at: https://pdxscholar.library.pdx.edu/honorstheses

Let us know how access to this document benefits you.

\section{Recommended Citation}

Powell, Madeline, "A Picture is Worth a Thousand Words: a Content Analysis of Self-Inflicted Face-ism on Instagram" (2016). University Honors Theses. Paper 349.

https://doi.org/10.15760/honors.340

This Thesis is brought to you for free and open access. It has been accepted for inclusion in University Honors Theses by an authorized administrator of PDXScholar. Please contact us if we can make this document more accessible: pdxscholar@pdx.edu. 
Portland State University

PDXScholar

University Honors Theses

University Honors College

\section{A Picture is Worth a Thousand Words: A Content Analysis of Self- Inflicted Face-ism on Instagram}

Madeline N. Powell

Let us know how access to this document benefits you.

Follow this and additional works at: http://pdxscholar.library.pdx.edu/honorstheses

This Thesis is brought to you for free and open access. It has been accepted for inclusion in University Honors Theses by an authorized administrator of PDXScholar. For more information, please contact pdxscholar@pdx.edu. 
Running head: SELF-INFLICTED FACE-ISM ON INSTAGRAM

A Picture is Worth a Thousand Words:

A Content Analysis of Self-Inflicted Face-ism on Instagram

by

Madeline Powell

An undergraduate honors thesis submitted in partial fulfillment of the

requirements for the degree of

Bachelor of Science

in

University Honors

and

Communication

Thesis Adviser

Dr. Lauren Frank

Portland State University

2016 


\begin{abstract}
The purpose of this study was to explore potential gendered stereotypes by examining the facial prominence of male and female celebrities' social media images. The face-ism theory evaluates the facial prominence of a depiction; higher facial prominence prompts viewers of media to assume the person they are observing is more intelligent and more likeable in general. In previous research men had higher facial prominence than women, which calls in to question the perpetuation of gendered stereotypes in media. This research is a content analysis of 600 selfselected Instagram photos, posted by the top 30 followed men and women celebrities on Instagram. The images were analyzed to test differences in facial prominence using the face-ism index to measure the head-body ratio of the celebrities. The popularity of the images and the occupation of the celebrity were also coded. The results of this study found no difference in facial prominence between men and women, suggesting that celebrities' self-selected images do not replicate the gender-biased facial prominence seen in images selected in marketing, advertising, and film. However, facial prominence for both female and male celebrities was very low compared to other studies. This study also found that occupation and popularity have no relationship to the level of facial prominence. The implications for new media in regard to the face-ism theory should be explored further in additional research.
\end{abstract}

Keywords: face-ism, gender representation, Instagram, celebrities, social media 


\section{A Picture is Worth a Thousand Words:}

\section{A Content Analysis of Self-Inflicted Face-ism on Instagram}

For centuries, the study of gender representations has fixated many scholars and led to a large body of gender-centered research. The difference in the way that men and women are depicted in media content makes up a large portion of that research (Archer, Iritani, Kimes, \& Barrios, 1983; Konrath, \& Schwarz, 2007; Copeland, 1989; Costa, \& Bitti, 2000; Levesque \& Lowe, 1999). A common finding is that women are portrayed in very stereotypical roles and are often objectified (Smith \& Cooley, 2012). Although this finding is not new, women are traditionally underrepresented and even when they are represented, these depictions may be significantly different than representations of men (Schwarz \& Kurz, 1989). Research has brought the level of facial prominence in images of men and women to attention. Men are typically depicted with high facial prominence and a focus on men's intellect and personality as opposed to women's depictions, which are usually focused on the whole body and highlight that women's physical attractiveness (Schwarz et al., 1989). The purpose of this study is to explore potential gendered stereotypes by examining the facial prominence of male and female celebrities' social media images.

This research will build upon previous work done by communication scholars on the theory of face-ism, originally explored by Archer et al. (1983). Face-ism, also known as facial prominence (the relative prominence of the face in an image of men and women), describes the tendency for depictions of men to have greater facial prominence than depictions of women (Archer et al., 1983). An especially interesting concept pertaining to high and low facial prominence in the media is the conclusions that people draw based on how much of a subject's head they see versus how much of the subject's body they see. Higher facial prominence prompts 
viewers of media to assume the subject they are observing is not only more intelligent, but also more "likeable" (Smith et al., 2012). One problematic conclusion that has been drawn from faceism research is that women are much more likely to be represented with low facial prominence, and thus put in the category of less intelligent and less likeable (Schwarz \& Kurz, 1989). This finding leaves our society with a very pressing question: "how do representations support or refute stereotyped conceptions of men and women, and do differences with regard to facial prominence support a gender stereotype that we might not want passed to subsequent generations" (Mathews, 2007, p. 525)?

In order to look at representations of men and women in today's society, I will examine celebrities' posts on Instagram and code for facial prominence and likeability. Social media and other new media technologies have become a central part of everyday interaction (Colapinto \& Benecchi, 2014), and society often promotes gender role markers and social norms through photographs and other visual displays (Rose, Makey-Kallis, Shyles, Barry, Biagini, Hart, \& Jack, 2012). Instagram, for example, is a social media platform that allows users to display mainly visual content. Malik, Dhir, and Nieminen (2015) explain that sharing photos online is an extremely popular activity and can help fill people's social interaction needs by providing them an outlet for self-expression, self-presentation, communication, and maintaining social relationships. Social media is used heavily by our society and may provide an accurate reflection of how men and women are represented in Western culture and also how they represent themselves, which I will elaborate on in the literature review.

\section{Celebrities and Instagram}

Celebrities can have a large impact on society and the way that people view society. Celebrities are characterized by the positive emotional response that are derived from the actors' 
positive influence on the audience and their potential to fulfill certain behavioral goals including, satisfying a need for gossip, fantasy, identification and attachment (Colapinto \& Benecchi, 2014, p. 221). Smith and Sanderson (2015) note that social media is commonly used by people who are in the spotlight to share aspects of their personality and identity that are overlooked in mainstream media. This allows those in the spotlight to control the content that is posted on their social media platforms and essentially be in control of their self-presentation. Athletes, actors, singers, and models that are well known are often categorized as celebrities.

Adolescents often form secondary attachments to figures they encounter in the media; these relationships can carry over into adulthood and influence how people shape their identity and feelings of self-worth (Boone \& Lomore, 2001). Boone \& Lomore (2001) found young adults were moderately to strongly attracted to media figures that they had identified as idols in their lives and this attachment could significantly shape identity development. Additionally, Blumler and Gurevitch (1974) found that certain people tend to form emotional attachments called parasocial relationships with media personalities (Griffin, 2012). Essentially, the media viewer becomes emotionally attached and feels a sense of friendship to the media personality or "celebrity." Parasocial relationships can sometimes result in the media viewer reproducing the actions of his or her celebrity idol (Griffin, 2012).

This study includes celebrities in the sample partly due to their influence on our culture, but also the availability of the visual and verbal content that is on their social media profiles. In this research, celebrities are defined as anyone that is in the public eye and has a large following base, particularly on Instagram. Instagram is one of the fastest growing social media platforms (Lunden, 2014) and has over 300 million monthly active users (HootSuite, 2016). Instagram provides users with a visual platform to upload images and content, on average users share 70 
million photos per day. Due to the nature of this research, Instagram is the perfect visual platform for image examination.

\section{Gender Stereotypes and Media}

Stereotypes are "beliefs that hold that all members of a group share the same set of characteristics, attitudes, or life conditions" (Smith et al., 2012, p. 280). Stereotypes can be applied to groups and individuals based on characteristics like gender, race, attitudes, or life conditions. Stereotypes can be extremely harmful to individuals and communities. Bandura (2009) states that depictions may have real life consequences for perceptions of men and women. Depictions of men and women often reflect societal norms and attitudes and have the potential to perpetuate common gender stereotypes. Collins (2011) notes that women are not only underrepresented in the media, but when women are represented they are often sexualized, shown in traditionally feminine rolls and "subordinated in various ways, as indicated by their facial expressions, body positions, and other factors" (p. 290). The current study is particularly interested in the body position (facial prominence) of men and women in social media.

Traditionally, stereotypical male traits like dominance and intelligence are associated with one's head. This is said to be true because people associate the head with the brain, and in turn intelligence (Smith et al., 2012). Certain stereotypical female traits such as expressiveness and warmth are associated with the body (Levesque et al., 1999). The perpetuation of these stereotypes in the media may be affected by the tendency for women's bodies to be more prominent than their heads and vice versa for men.

\section{Face-ism Theory}

Archer et al. (1983) coined the concept of face-ism, which evaluates the facial prominence of a depiction; facial prominence is assessed by a face-ism index that measures the 
ratio of distance from the top of someone's head to the lowest point of their chin to the distance from the top of the head to the lowest visible part of the body (Schwarz et al., 1989). Archer et al. (1983) conducted five studies to test sex differences in facial prominence. The first three studies indicated that there was a "consistent asymmetry in the images of the sexes in published photographs across several cultures and artwork across several centuries" (p. 733). The fourth study found that the difference in facial representations of men and women is also seen in casual drawings. The fifth and final study they conducted drew some very interesting conclusions; the same person is perceived more favorably when their face is prominent versus when it is not. Since these original studies were completed, the high facial prominence of men in media depictions has been seen not only across multiple cultures, but time periods as well (Levesque \& Lowe, 1999). In a study done by Copeland (1989), the framing of men and women in a sample of primetime TV commercials and programs was examined. Copeland (1989) found that face-ism was present in TV as well as well as other mediums; men were shot at a closer perspective than women.

A study done by Schwarz et al. (1989) pertaining to the impact that facial prominence has on impression formation reflected a global positive effect of high facial prominence. Male and female subjects were presented with either full body or portrait style photographs of men and women. People with a high (portrait) rather than low (full body) degree of facial prominence were evaluated as more intelligent, assertive, and ambitious. People with higher facial prominence were also rated as more likeable and expressive. Overall, a high degree of facial prominence prompts more positive rather than sex-typed attributions, but men typically are portrayed with higher facial prominence than women, leading to the assumption that men will be viewed as more competent than women more often (Schwarz et al., 1989). 
Following the study done by Schwarz et al. (1989) on stereotypes, Levesque et al. (1999) extended their research to explore the effects that facial prominence has on interpersonal perception by presenting subjects with videotapes of men and women with various levels of facial prominence. In the absence of other information, videotaped stimuli led subjects to form opinions and impressions based on the facial prominence of subjects. This means that without any other information, people form opinions merely by evaluating facial prominence. Similarly, Levesque et al. (1999, p. 255) found that high facial prominence resulted in more favorable ratings than low facial prominence in the settings with occupational information (Levesque et al., 1999, p. 255). They also found that women, people of color, and people in particular occupations are typically shown with less facial prominence and are evaluated less favorably than men, people of European descent, and people in occupations with high facial prominence. This means that face-ism may actually prompt a particular stereotype. Finally, they found a general positivity bias for people with higher facial prominence because people pictured with high facial prominence appear more proximal, meaning there is less distance between the subject of the image and the person judging it (Levesque et al., 1999, p. 256).

Even though there is evidence that supports face-ism, there is some evidence that contradicts it. Sparks and Fehlner (1986) conducted a study in which they compared gender depictions in magazines using the face-ism index. They coded 292 photographs from the 1984 election of the four presidential campaign candidates in the U.S. They found that the female vice president (G. Ferraro) had a similar degree of facial prominence to her male counterparts (Sparks et al., 1986). In a second study, photographs of female and male journalists and government officials were evaluated for facial prominence using the face-ism index as well. The results from 
this study also contradicted the findings by Archer et al. (1983) by suggesting that men do not have higher facial prominence than women in images.

In 2007, Konrath and Schwarz conducted a study in which photographs retrieved from official government websites of all of the U.S. Governors, Senators, Representatives and Members of Parliament in Canada, Australia, and Norway were analyzed for facial prominence. In all of the countries, male politicians were shown with higher facial prominence than female politicians. This finding supports the hypothesis of face-ism, while contradicting the findings of Sparks et al. (1986), where male politicians were not represented with higher facial prominence than female politicians. The main findings of the study suggest that there is no actual gender difference in body proportions (women's heads are actually larger relative to the size of their bodies) (Konrath et al., 2007). They also found that overall male politicians have more prominent faces in the portraits than the female politicians, in the U.S. Additionally, Hispanic politicians usually presented themselves with lower facial prominence than African American politicians who showed more facial prominence than any other group.

Mathews (2007) studied depictions that were not self-selected by people in certain occupations (e.g. executive/ businessperson, sports figure, actor/entertainer, model, politician, scientist/educator, or unclassifiable), and found that individuals depicted in intellectually focused occupations had higher face-to-body rations than those depicted in physically focused occupations. In addition to that, women in the same intellectually focused roles as men had lower face-to-body ratios to men. Similarly, women in physical occupations such as sports figures or models had higher face-to-body ratios than men. This study indicated there is a discrepancy in the way in which men and women are portrayed in print media, even when they have similar occupational status. Mathews (2007) notes that "somewhere during the photo- 
graphic/layout/editing process in popular magazines, men are differentially highlighted for their cerebral qualities when being depicted in intellectual occupations, and differently highlighted for their physical qualities when being depicted in physical occupations; women's cerebral and somatic qualities are not similarly highlighted" (p. 523).

Smith and Cooley (2012) examined self-selected photographs and user-provided information of 1400 Facebook (and other similar sites in other countries) profiles of men and women, ages 25 and under, from the United States, Ghana, Australia, The Republic of Korea, France, Brazil, and Russia. Smith et al. (2012) used the face-ism index to measure the headbody-ratio of their subjects and coded for gender, age, ethnicity, and number of friends on social media. The purpose of their research was to "highlight the shared cultural commonalities and distinctions of users of social networking sites across the globe" (Smith et al. 2012, p. 493). The results from the study showed that men had significantly higher facial prominence than women in most countries, even though the photographs on the social media sites were self-selected (Smith et al., 2012). Smith et al. (2012) conclude that the results from this study suggest a significant internalization of long-held stereotypes.

\section{Contrast of Women as Objects}

The objectification of women in the media goes with gender stereotypes. Objectification has been explored in depth and is pretty easy to recognize. The face-ism theory on the other hand examines a hidden gender discrepancy in physical depictions. The face-ism index provides researchers with an objective way to look at gender discrepancies in the media than simply coding an image by its potential objectification status. Typically, a picture conveys many messages: the gender of the person, the role they are playing, and many other key elements that help us assess that person's character. Essentially, an image is worth a thousand words. Gender 
differences in the mass media have been examined and results consistently find that women are still portrayed in background roles, in comparison to men (Smith et al., 2012). Along with the portrayal of women in background roles, they are also largely displayed in the media with the main focus being on their body. Body-centered imagery is said to show that women remain largely stereotyped as unintelligent, attractive bodies with no personality (Matthews, 2007). In a photograph that is acting to objectify a person, the role that they are playing in that image, whether it is a stereotypical role or something else, is usually highlighted as the main takeaway. It should be noted that objectification is extremely detrimental; research by Lougnan, Haslam, Murnane, Vaes, Reynolds, and Suitner (2010) indicated that when people are objectified they are denied personhood. When examining a photograph based on the face-ism index, the potential stereotypes are much harder to identify or considered latent sexism. The main determinant of the sexism in the photograph is not determined by the role that the subject in the image is playing, but by the prominence of their head versus their body. Even when stereotypical roles change or the social rules of sexism vary by different cultures, the prominence of someone's face can always be measured and this is why the face-ism index is explored further as an objective measurement.

Lower facial prominence of women is even seen in self-selected pictures, meaning the women chose to share these images (Smith et al., 2012). It seems unlikely that someone would consciously share an image that she felt would devalue her. It is interesting to acknowledge that even in self-selected images, the level of facial prominence is lower for women. This study will look at images that celebrities have selected to post publicly. This study was designed to build on previous research concerning the face-ism index in relation to gender, occupation, and popularity in an online setting. In order to do this, I am posing the following research questions. 
RQ1: Does facial prominence vary by gender?

RQ2: What is the relationship between level of facial prominence in social media posts and the popularity of the picture?

RQ3: Does facial prominence vary by occupation?

\section{Method}

\section{Sample}

This study examined the photographs and user-provided information on celebrities' Instagram accounts. Celebrities are essentially known for being known. The celebrities I used in this sample were categorized by occupation. These occupations include: TV personality, athlete, singer, and model. The sample included 30 of the most followed celebrities on Instagram as of May 2016. Since there were a significantly higher number of female celebrities with more followers, some were excluded from the sample in order to get an even amount of men and women (15 women, 15 men). It should be noted that one of the original celebrities selected for the study, Justin Bieber, deleted his Instagram account prior to the collection of data and was replaced with another male celebrity, Dan Bilzerian. Celebrities were used as the sample due to their cultural and social influence in the world as well as ease of access to their profiles. Twenty photographs were assessed per celebrity, totaling 600 images. The subjects in the photos were then closely examined to determine the level of facial prominence and the popularity of each picture.

Some of the inclusion rules for photo selection used in this research were first proposed by Archer et al. 1983), and used in subsequent studies (Smith et al., 2012). I also added certain criteria specific to the sample that I am using. First, only photographs with one human subject in them were selected. Second, the photograph was of the celebrity being evaluated. Third, videos 
of the subject were not used, since this study was tailored specifically to images. Finally, images with a co-subject (posing with an animal, a person driving in a car etc.) were excluded.

Images were included even if there was another person in the image, as long as that person(s) feature's (i.e. nose, lips, eyes) were blurred enough to be unrecognizable and were not the focal point of the image. Images were also included even if there was a painting/picture of another person or thing in the image with the main celebrity. Images that only showed the celebrities' hand or foot were included, and counted as a face-ism index of 0 (no face in the image). Pictures with filters such as black and white, sepia, lo-fi, etc. were still included as well. Images where the celebrity was on/in a magazine were still included. Images were excluded if it was too hard to identify the celebrity that was being measured, if it showed the celebrity at a much younger age, or if the subject in the image was too hard to identify as the celebrity.

\section{Coding Criteria}

Once the data was collected from Instagram by selecting the most recent 20 photographs that met the inclusion criteria, the pictures were measured using the face-ism index. The face-ism index measures the facial prominence of a given person in a photo. As noted by Archer et al. (1983):

The method consists of creating an index that is the ratio of two linear measurements. The numerator of this index is the distance (in millimeters or any other unit) in a depiction from the top of the head to the lowest point of the chin. The denominator of the index is the distance from the top of the head to the lowest visible part of the subject's body. (p. 726)

The index of facial prominence is then calculated by dividing the first measurement by the second. The index can measure from .00 (the face is not shown at all) to 1.00 (the picture shows only the face and no other part of the body). Figure 1 shows the method used to measure images. The higher an index number is, the higher the facial prominence of a picture; and therefore, the lower the index number, the lower the facial prominence. Following the coding criteria used by 
Archer et al. (1983) and subsequent studies (Smith et al., 2012), in situations where a subject's face was covered by an object, such as a beard or a hat, the location of the boundaries were estimated.

The measurement process was completed using a computer program called Bluebeam. The measurements used to find the facial prominence index for each picture were drawn on the photograph and then I recorded the values of each measurement and calculated the indexes accordingly. After each picture had been measured for the facial index value, the facial index averages for gender and occupation were compared.

From the selected Instagram pictures, facial prominence, gender, and likeability were the main variables of interest. Popularity was operationalized by the number of likes a picture had. The coder used the self-identified gender of the celebrities as the measurement for gender. The number of followers an individual had was coded as a raw number.

\section{Results}

Overall, 30 celebrities were selected to be analyzed (50\% males, 50\% female). Of the 30 celebrities, only 7 were athletes $(23 \%)$. As seen in Table 1 , the four occupations identified were TV personalities/actors, models, singers and athletes. For example, Kim Kardashian is a TV personality who is mainly known for being a reality TV star, which is why she fits in the TV personality category. Unfortunately, there were no female athletes in the selection of celebrities, which was based on popularity. The average face-ism indexes for the various occupations were extremely similar to each other. It should be noted that it was quite hard to collect images for this study that fit the inclusion criteria. In some cases, in order to find 20 pictures for each study it was required to go back almost two years in time. More often than not this was caused because there were multiple people in the images posted. 
The average face-ism index for all of the celebrities combined was $0.28(S D=0.20)$. This is a very low level facial prominence. The average face-ism index for other studies is much higher than the average indicated in this study. Konrath et al. (2007) found women and men to both have a higher face-ism index than 0.75 in their study on American politicians. Copeland et al. (1989) found that both men and women scored higher than a 0.41 face-ism index on primetime entertainment. Finally, Archer et al.'s (1989) result's showed that the average face-ism index for women in ads was 0.41 and men's was 0.55 . The celebrity with the highest average face-ism index in this study was Vin Diesel (0.48) followed by Katy Perry (0.41). Nicki Minaj had the lowest average face-ism index across her 20 photos $(0.20)$.

RQ1 asked if facial prominence varied by gender. The findings of an independent samples $t$-test contradicted the hypothesis proposed by the face-ism theory that men would have much greater facial prominence. The overall mean face-ism index for men $(M=0.28, S D=0.20)$ was extremely similar to the mean face-ism index for women $(M=0.29, S D=0.19)$ unlike the predictions of the face-ism theory, $t(598)=0.23, p=.49$. The test shows that men and women's face-ism index in Instagram pictures were almost exactly the same.

RQ2 asked if there would be a relationship between level of facial prominence in social media posts and the popularity of the picture. There was no correlation in the data set between level of facial prominence and the popularity of the picture (Spearmen $r_{s}=0.04, p=.38, N=$ 600). This test shows that there is no relationship between level of facial prominence and the amount of likes that a picture has.

RQ3 asked if facial prominence in celebrities' Instagram pictures would vary by occupation. The two occupations tested were athletes $(M=0.28, S D=0.20)$ and other $(M=0.29$, 
$S D=0.19)$, which encompasses TV personalities, models, singers, etc. There was little difference in facial prominence by occupation, $t(598)=0.19, p=.15$.

\section{Discussion}

The purpose of this research was to explore potential gendered stereotypes by examining the facial prominence of male and female celebrities' social media images. The three research questions addressed in this study were: does facial prominence vary by gender, what is the relationship between level of facial prominence in social media posts and the popularity of the picture, and does facial prominence vary by occupation.

In other research studies on face-ism, the tendency for men to have higher facial prominence in pictures is much greater than that of women. This is seen especially often in images that have been chosen for people like ads, or magazine covers. Previous research shows evidence of gender display biases, not only across cultures, but through different time periods as well. Schwarz et al. (1989) found the media's face-ism bias likely contributes to a perception of men as more competent than women, due to this difference in gender displays (men with higher facial prominence and women with lower facial prominence). This study was unique in that it looked at celebrities' self-selected images to see if they replicate the gender-biased facial prominence examined in other studies. This study found that the gender display biases identified in other studies do not exist in self-selected Instagram photos of celebrities.

It seems that overall, facial prominence was very low among all celebrities, regardless of gender or occupation. This could be because Instagram is an informal platform, unlike LinkedIn, which is directed towards business professionals and thus encourages headshots. The sample of celebrities is also likely to have impacted the average face-ism index. Most likely there are 
norms on Instagram that the top celebrities all follow, which in turn helps them keep a large amount of followers.

Overall facial prominence could also be low because the "selfie" picture is considered narcissistic and conceited in our society. In this regard, higher facial prominence could come off as less likeable because the subject of the image is perceived as "vain" or "self-centered." While selecting photos to be included in the study, I found that many celebrities have more images of themselves with other people or other subjects, like a dog or cat.

Cooley et al. (2012) noted "the theory of face-ism claims the embodiment of a man is in the face and head; yet nations with the most masculine cultural orientations present themselves with the least facial prominence" (p. 292). In their study, nations such as the United States, Australia, and Russia, all nations with high masculinity, were found to have the lowest face-ism scores (Cooley et al., 2012). It is possible that characteristics of masculinity are best conveyed in photographs that show more of the body and torso, which gives a view of clothes, personal wealth, and occupation. Instagram is a platform where brands pay celebrities to endorse their company and upload images that have their product in it. Future research could also code for whether or not the image was sponsored by a company. It may also be the case that preconceived stereotypes are starting to diminish on the online environment. However, exploitation of one's body, regardless of gender, may be increasing. Images with lower facial prominence may allow celebrities to include an article of clothing or object that they are being asked to promote. There are magazines that typically do show face-ism by gender. Mathew's (2007) findings suggest that photographers, editors, and people in the position to dictate how individuals are depicted in magazines, might place differential facial priority on men and women with regard to occupational status. 
There was no correlation between face-ism and likes. Schwarz et al. (1989) reported that a high degree of facial prominence elicits more positive attributions in general (p. 316). These positive attributions included perceptions of the subject being active, intelligent, assertive, and independent. This study aimed to test the assumption that images with higher facial prominence are more likely to elicit more positive attributions by comparing the amount of likes a picture received with the level of facial prominence in the picture. The findings suggest there is no correlation between level of facial of prominence and positive attributions, at least in this online context. I expected the images with higher facial prominence to receive more likes. This was not the case and it opens up a conversation about how celebrities should select images if their goal is to receive more likes. Maybe Instagram is a platform where high facial prominence doesn't change the way that people interact with images; it only changes the way that you feel about a person. For example, images of celebrities with low facial prominence still receive a large of amount of likes, but it is hard to know whether or not someone thinks of those people as more intelligent or less intelligent after they view the picture. All we know is that the level of facial prominence in an image is not influencing the number of likes.

Finally, this study looked into the relationship between level of facial prominence and the occupation of the celebrity. This too came back with no relationship. In a study done by Mathews (2007), individuals depicted in intellectually focused occupations had higher face-tobody rations than those depicted in physically focused occupations. The argument could be made that all of the celebrities included in this study are associated with more physically focused occupations (athletes, models, singers, etc.). This could explain the generally low face-ism index. Unfortunately, there are no top followed celebrities that are depicted in more intellectually focused occupations. 
All in all, there are many reasons that could explain why the facial prominence of celebrities is much lower than the face-ism theory would suggest it be. Maybe our culture is moving to a time where intelligence and likeability are not associated with facial prominence anymore (at least in this demographic), but through the wealth and confidence conveyed by showing off one's body. Assumptions should not be made though and future research could look into the effects of these types of photos on likeability, intelligence, and other perceptions. The hierarchy of our society or socio-economic status may be more important for celebrities to convey than their intellect. This is typically accomplished by showing off expensive clothes and accessories, best seen in images of the full body.

\section{Limitations and Future Research}

Although this study does have limitations, it is an attempt to fill that gaps that have been identified in previous face-ism research. The biggest limitation of the study is related to the accessibility of Instagram pictures and therefore a diverse sample of people. Although the group selected for this research provides a representation of our society, it does not accurately reflect the general population. The sample could have also been larger, both in terms of celebrities selected and the amount of images measured for per each celebrity.

Another limitation identified is a lack of diversity among physical and intellectual occupations. All of the celebrities selected for this study are known for occupations that are mainly physical in nature (i.e., athletes, singer, models, etc.). The inclusion of people that fit into more traditional "intellectual occupations" (i.e., lawyer, doctor, professor, etc.) could have provided the study with insight into how people in those occupational categories represent themselves in images. The inclusion challenges of this study could also be considered a limitation. In most cases, it was hard to find recent images that matched the inclusion criteria 
above. This limitation also relates to what future research could address. It may be time to update the inclusion criteria used in face-ism research in order to get a more representative sample.

Future research could focus more on the psychology behind self-selected images, or why the user is representing themselves with higher or lower facial prominence. This could lead to insight around why these images were selected.

\section{Conclusion}

Higher facial prominence prompts viewers of media to assume the subject they are observing is not only more intelligent, but also more "likeable." This presents a problematic issue in our society because women are more often than men portrayed with lower facial prominence in film, advertising, and marketing. Gender stereotypes and unrealistic representations of women continue to be present in popular culture, yet the results from this study contradict the gender differences pointed out in prior research on facial prominence. That being said, it is difficult to know if gendered stereotypes continue, or if they have just changed. There are certain social media norms on Instagram that users follow in order to gain followers and get more likes on their images. Some of these norms include showing more of ones body. Smith and Sanderson (2015) note that a selfie, or headshot, is often limiting and focuses solely on the subject of the photograph. Context can be lost in these types of images, and can go against what people are trying show in their pictures, especially celebrities. Some of these Instagram norms may explain the lower facial prominence all around. 


\section{References}

Archer, D., Iritani, B., Kimes, D. D., \& Barrios, M. (1983). Face-ism: Five studies of sex differences in facial prominence. Journal of Personality and Social Psychology, 45(4), 725-735. http://doi.org/http://dx.doi.org/10.1037/0022-3514.45.4.725

Boon, S., \& Lomore, C. (2001). Admirer-celebrity relationships among young adults. Human Communication Research, 27(3), 432-465. http://doi.org/10.1111/j.14682958.2001.tb00788.x

Cheek, N. N. (2016). Face-ism and objectification in mainstream and LGBT magazines. PLOS ONE, 11(4), e0153592. http://doi.org/10.1371/journal.pone.0153592

Colapinto, C., \& Benecchi, E. (2014). The presentation of celebrity personas in everyday twittering: managing online reputations throughout a communication crisis. Media, Culture \& Society, 36(2), 219-233. http://doi.org/10.1177/0163443714526550

Collins, R. L. (2011). Content analysis of gender roles in media: Where are we now and where should we go? Sex Roles, 64(3-4), 290-298. http://doi.org/10.1007/s11199-010-9929-5

Copeland, G. A. (1989). Face-ism and primetime television. Journal of Broadcasting \& Electronic Media, 33(2), 209-214.

Costa, M., \& Bitti, P. E. R. (2000). Face-ism effect and head canting in one's own and others' photographs. European Psychologist, 5(4), 293-301. http://doi.org/10.1027//10169040.5.4.293

Griffin, E. (2012). A First Look at Communication Theory ( $8^{\text {th }}$ Edition). Boston, MA: McGrawHill.

Hall, C. C. I., \& Crum, M. J. (1994). Women and "body-isms" in television beer commercials. Sex Roles, 31(5-6), 329-337. http://doi.org/http://dx.doi.org/10.1007/BF01544592 
Hootsuite. (2015, September). A long list of instagram statistics and facts that prove its importance. Retrieved from https://blog.hootsuite.com/instagram-statistics-for-business/ Instagram: most-followed accounts worldwide. (2016, May). Retrieved from http://www.statista.com/statistics/421169/most-followers-instagram/

Katz, E., Blumler, J., \& Gurevitch, M. (1973). Uses and gratifications research. The Public Opinion Quarterly, 37(4), 509-523. doi:10.1086/268109

Konrath, S. H., \& Schwarz, N. (2007). Do male politicians have big heads? Face-ism in online self-representations of politicians. Media Psychology, 10(3), 436-448. http://doi.org/10.1080/15213260701533219

Lauricella, A. R., Cingel, D. P., Blackwell, C., Wartella, E., \& Conway, A. (2014). The mobile generation: Youth and adolescent ownership and use of new media. Communication Research Reports, 31(4), 357-364. http://doi.org/10.1080/08824096.2014.963221

“Leading social networks worldwide as of May 2016, ranked by number of active users" (2016). Statista. Retrieved from http://www.statista.com/statistics/272014/global-socialnetworks-ranked-by-number-of-users/

Levesque, M. J., \& Lowe, C. A. (1999). Face-ism as a determinant of interpersonal perceptions: The influence of context on facial prominence effects. Sex Roles, 41(3-4), 241-259. http://doi.org/10.1023/A:1018806329197

Loughnan, S., Haslam, N., Murnane, T., Vaes, J., Reynolds, C., \& Suitner, C. (2010). Objectification leads to depersonalization: The denial of mind and moral concern to objectified others. European Journal of Social Psychology, 40(5), 709-717. http://doi.org/10.1002/ejsp.755 
Lunden, I. (2014, January). Instagram is the fastest-growing social site globally, mobile devices rule over PCs for access. Retrieved from http://techcrunch.com/2014/01/21/instagram-isthe-fastest-growing-social-site-globally-mobile-devices-rule-over-pcs-for-social-access/

Matthews, J. L. (2007). Hidden sexism: Facial prominence and its connections to gender and occupational status in popular print media. Sex Roles: A Journal of Research, 57(7-8), 515-525. http://doi.org/http://dx.doi.org/10.1007/s11199-007-9276-3

McArthur, L. Z., \& Resko, B. G. (1975). The portrayal of men and women in american television commercials. Journal of Social Psychology, 97(2), 209.

Perloff, R. M. (2015). Mass communication research at the crossroads: Definitional issues and theoretical directions for mass and political communication scholarship in an age of online media. Mass Communication and Society, 18(5), 531-556. http://doi.org/10.1080/15205436.2014.946997

Quinn, K. (2016). Contextual social capital: Linking the contexts of social media use to its outcomes. Information, Communication \& Society, 19(5), 582-600. http://doi.org/10.1080/1369118X.2016.1139613

Reilly, D., \& Neumann, D. L. (2013). Gender-role differences in spatial ability: A meta-analytic review. Sex Roles, 68(9-10), 521-535. http://doi.org/http://dx.doi.org/10.1007/s11199013-0269-0

Rose, J., Mackey-Kallis, S., Shyles, L., Barry, K., Biagini, D., Hart, C., \& Jack, L. (2012). Face it: The impact of gender on social media images. Communication Quarterly, 60(5), 588607. http://doi.org/10.1080/01463373.2012.725005

Schwarz, N., \& Kurz, E. (1989). What's in a picture? The impact of face-ism on trait attribution. European Journal of Social Psychology, 19(4), 311-316. 
Smith, L. R., \& Cooley, S. C. (2012). International faces: An analysis of self-inflicted face-ism in online profile pictures. Journal of Intercultural Communication Research, 41(3), 279296. http://doi.org/10.1080/17475759.2012.728771

Smith, L. R., \& Sanderson, J. (2015). I'm going to Instagram it! An analysis of athlete selfpresentation on Instagram. Journal of Broadcasting \& Electronic Media, 59(2), 342-358. http://doi.org/10.1080/08838151.2015.1029125

Sparks, G. G., \& Fehlner, C. L. (1986). Faces in the news: Gender comparisons of magazine photographs. Journal of Communication, 36(4), 70-79. http://doi.org/http://dx.doi.org/10.1111/j.1460-2466.1986.tb01451.x

“Top 100 Instagram Profiles by Followers” (2016, May). Socialblade Instagram Statistics. Retrieved from https://socialblade.com/instagram/top/100/followers

Tripathy, J. (2010). How gendered is gender and development? Culture, masculinity, and gender difference. Development in Practice, 20(1), 113-121. http://doi.org/10.1080/09614520903436901 
Figure 1. Image of Selena Gomez with face-ism measurements

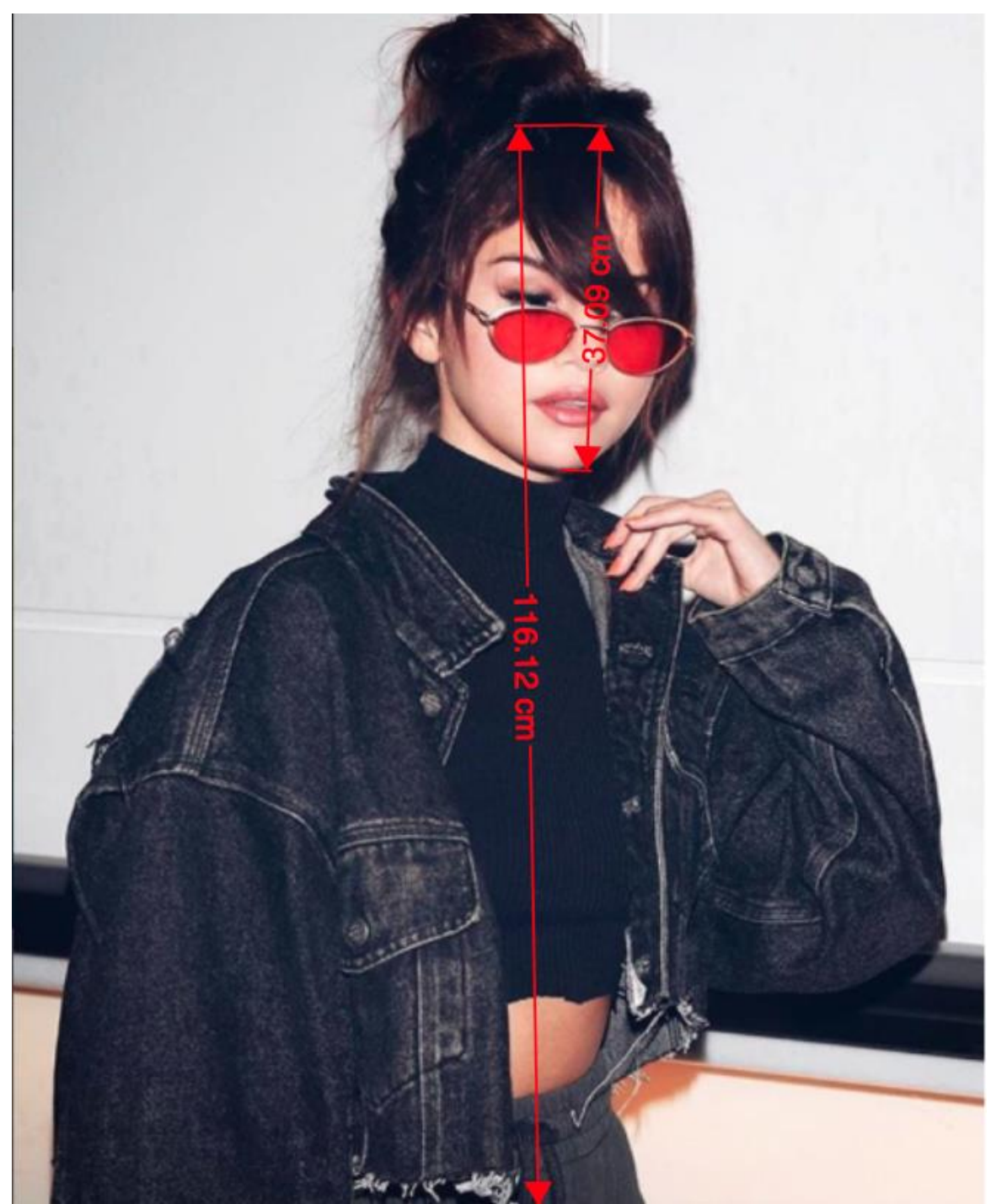

Figure 1. Sample image of Selena Gomez showing the method used to measure the images in this study. Measurement 1 is from the top of the head to the chin, measurement 2 is from the top of the head to the lowest visible part of the subject's body. To find the face-ism index the first measurement is divided by the second. 


\section{Table 1}

List of top followed celebrities (15 men; 15 women) on Instagram with the average face-ism index for each

\begin{tabular}{cllllc}
\hline Rank & Gender & Celebrity & Occupation & Followers & Face-ism Index \\
\hline 2 & F & Selena Gomez & Singer & $97,782,519$ & 0.22 \\
3 & F & Taylor Swift & Singer & $90,515,222$ & 0.31 \\
4 & F & Beyonce & Singer & $83,637,956$ & 0.27 \\
5 & F & Ariana Grande & Singer & $83,368,385$ & 0.35 \\
6 & F & Kim Kardashian & TV/Actor & $81,514,236$ & 0.22 \\
8 & M & Cristiano Ronaldo & Athlete & $75,711,783$ & 0.31 \\
9 & F & Kylie Jenner & TV/Actor & $73,715,046$ & 0.25 \\
10 & F & Nicki Minaj & Singer & $65,348,662$ & 0.20 \\
11 & M & Dwayne Johnson & TV/Actor & $64,888,570$ & 0.22 \\
12 & F & Kendall Jenner & Model & $64,797,271$ & 0.30 \\
15 & M & Neymar & Athlete & $58,523,388$ & 0.33 \\
16 & F & Khloe Kardashian & TV/Actor & $55,559,368$ & 0.27 \\
17 & F & Katy Perry & Singer & $55,373,076$ & 0.41 \\
18 & M & Leo Messi & Athlete & $53,278,080$ & 0.29 \\
19 & F & Miley Cyrus & Singer & $51,179,785$ & 0.40 \\
20 & F & Jennifer Lopez & Singer & $51,043,021$ & 0.36 \\
21 & F & Kourtney Kardashian & TV/Actor & $45,805,406$ & 0.21 \\
23 & F & Demi Lovato & Singer & $45,599,086$ & 0.27 \\
24 & F & Rihanna & Singer & $43,592,582$ & 0.29 \\
25 & M & Kevin Hart & TV/Actor & $41,421,114$ & 0.21 \\
28 & M & Justin Timberlake & Singer & $34,732,422$ & 0.29 \\
33 & M & Chris Brown & Singer & $30,637,737$ & 0.21 \\
34 & M & David Beckam & Athlete & $28,338,865$ & 0.26 \\
35 & M & Drake & Singer & $27,764,637$ & 0.25 \\
36 & M & Vin Diesel & TV/Actor & $26,256,966$ & 0.48 \\
37 & M & James Rodríguez & Athlete & $25,316,326$ & 0.27 \\
38 & M & Lebron James & Athlete & $24,686,513$ & 0.27 \\
42 & M & Gareth Bale & Athlete & $22,245,022$ & 0.24 \\
44 & M & Zac Efron & TV/Actor & $20,130,804$ & 0.30 \\
45 & M & Dan Bilzerian & TV/Actor & $19,168,313$ & 0.32 \\
\hline & & & & \\
\hline
\end{tabular}

Notes: Rank was determined by the number of followers each celebrity had in May 2016. The top followed brands or non-human accounts were excluded. The genders are represented by $\mathrm{F}$ for female and $\mathrm{M}$ for male. The average Face-ism index column represents the average face-ism indicated for each celebrity. 